\title{
Preservation and variation of ion-to-electron temperature ratio in the plasma sheet in geo-magnetotail
}

\author{
ChuXin Chen* \\ Chinese Academy of Sciences Key Laboratory of Geospace Environment, Department of Geophysics and Planetary Sciences, School of Earth and Space \\ Sciences, University of Science and Technology of China, Hefei 230026, China
}

\section{Key Points:}

- When IMF is southward, the reflection of electrons from the ionosphere, and subsequent multiple non-adiabatic accelerations at the reconnection site, are the primary reasons for the observed low ion-to-electron temperature ratio close to the earth at midnight.

- When IMF is northward, the reconnection in the nonlinear phase of the Kelvin-Helmholtz instability is the dominant process for solaroriginated plasma entering the Earth's magnetosphere.

- Turbulence on the boundaries of BBFs could change the temperature ratio in the far tail through the violation of the frozen-in condition of the ions.

Citation: Chen, C. X. (2021). Preservation and variation of ion-to-electron temperature ratio in the plasma sheet in geo-magnetotail. Earth Planet. Phys., 5(4), 337-347. http://doi.org/10.26464/epp2021035

\begin{abstract}
The ion-to-electron temperature ratio is a good indicator of the processes involved in solar wind plasma entering and being transported inside Earth's plasma sheet. In this study, we have demonstrated that patchy magnetic reconnection has the potential to preserve the ion-to-electron temperature ratio under certain conditions. If the charged particles are non-adiabatically accelerated no more than once in a single reconnection, the temperature ratio would be preserved; on the other hand, this ratio would not be preserved if they are accelerated multiple times. Consequently, under a northward interplanetary magnetic field (IMF) condition, the reconnection in the nonlinear phase of the Kelvin-Helmholtz instability is the dominant process for solar-originated plasma entering the Earth's magnetosphere, and the ion-to-electron temperature ratio is preserved inside the plasma sheet. When the direction of the IMF is southward, the reflection of electrons from the magnetic mirror point, and subsequent multiple non-adiabatic accelerations at the reconnection site, are the primary reasons for the observed low ion-to-electron temperature ratio close to the Earth at midnight. While reconnections that occur in the night-side far tail might preserve the ratio, turbulence on the boundaries of the bursty bulk flows (BBFs) could change the ratio in the far tail through the violation of the frozen-in condition of the ions. The plateau in the contour of the calculated ion-to-electron temperature ratio in the down tail distance between 40 and 60 Earth radii may explain the strong correlation between the ion and electron temperatures in the outer central plasma sheet, which has not been clearly understood till date.
\end{abstract}

Keywords: plasma sheet; ion-to-electron temperature ratio; patchy magnetic reconnection; non-adiabatic acceleration; bursty bulk flows; far tail

\section{Introduction}

The terrestrial magnetosphere acts as an impediment to the solar wind. Theoretically, the terrestrial magnetosphere should not contain solar plasma; however, it is actually the habitat of hot tenuous solar-originated plasma. Significant research has been conducted to determine how solar wind plasma enters the magnetosphere and how it is transported, once it is inside.

Among the parameters that are sensitive to the processes involved in the entry and transport of solar plasma, the ion-to-electron temperature ratio $\left(T_{\mathrm{i}} / T_{\mathrm{e}}\right)$ is one of the most important, and the preservation or variation of this ratio is a key indicator of the

Correspondence to: C. X. Chen, chuxin@ustc.edu.cn

Received 11 MAR 2021; Accepted 02 JUN 2021.

Accepted article online 08 JUN 2021.

(C)2021 by Earth and Planetary Physics. mechanism involved.

An analysis of the Active Magnetospheric Particle Tracer Explorers/lon Release Module (AMPTE/IRM) data by Baumjohann et al. (1989) indicated that the ion and electron temperatures were highly correlated $\left(T_{\mathrm{i}} / T_{\mathrm{e}}=7.2\right)$ in the outer central plasma sheet. In a case study using Cluster data, Lavraud et al. (2009) found that a low ion-to-electron temperature ratio $\left(T_{\mathrm{i}} / T_{\mathrm{e}} \approx 3\right)$ was preserved, as the plasma entered the magnetosphere. A statistical investigation of Time History of Events and Macroscale Interactions during Substorms (THEMIS) data (Wang CP et al., 2012) showed that beyond a distance of 40 Earth radii $\left(R_{\mathrm{e}}\right)$ in the tail, the $T_{\mathrm{i}} / T_{\mathrm{e}}$ ratio remained approximately constant from the magnetosheath to the cool plasma sheet.

On the other hand, Slavin et al. (1985) found that the $T_{\mathrm{i}} / T_{\mathrm{e}}$ ratio varied with the downtail distance. Their results indicated that $T_{\mathrm{i}} / T_{\mathrm{e}}$ 
was 7.8 over $30-60 R_{\mathrm{e}^{\prime}} 6.7$ over $60-100 R_{\mathrm{e}^{\prime}} 4.8$ over $100-210 R_{\mathrm{e}^{\prime}}$ and 5.7 beyond $210 R_{\mathrm{e}}$. Wang $C P$ et al. (2012) also analyzed the dependence of the $T_{\mathrm{i}} / T_{\mathrm{e}}$ ratio on the local time and radial distance from the Earth, and found that the ratio varied from approximately $6-10$, when the plasma sheet was relatively cool (i.e., during a northward IMF) to approximately 2-5 when it was relatively warm (i.e., during a higher auroral electrojet $(A E)$ value). In addition, based on a comparison of the specific entropies of the warm plasma between the distant- and near-Earth tails, Wang CP et al. (2012) suggested that non-adiabatic processes energized the electrons more than the ions, which would lead to a lower ratio of the warm plasma near the Earth at midnight compared to that in the distant tail.

The purpose of the present study is to ascertain whether there is a mechanism that preserves the $T_{\mathrm{i}} / T_{\mathrm{e}}$ ratio during the process of solar wind plasma entering into, and being transported within the plasma sheet. Because adiabatic processes preserve the ratio, we concentrate only on non-adiabatic processes. We have identified a mechanism that influences the above phenomenon and also determined the factors that can alter this ratio. The remainder of this paper is organized as follows. In Section 2, a process, which largely influences the entry of solar-originated plasma into the magnetosphere, is proposed. In Section 3, we explore the factors that can change the aforementioned ratio in the plasma sheet. Finally, a discussion is provided in Section 4, followed by a summary in Section 5.

\section{Entry of Charged Particles into the Magnetosphere}

Before conducting a detailed examination of every possible entry process, we first consider the variation of the $T_{\mathrm{i}} / T_{\mathrm{e}}$ ratio from the upstream solar wind to the magnetosheath, and subsequently from the magnetosheath to the magnetosphere.

Firstly, the ultimate source of energy of a charged particle, regardless of whether it is in the magnetosheath or magnetosphere, is the solar wind and not the magnetosheath or magnetosphere.

If the mass of a proton is the same as that of an electron, the $T_{\mathrm{i}} / T_{\mathrm{e}}$ ratio would always be preserved as solar-originated particles pass the bow shock and later on enter the magnetosphere. However, the mass of a proton is two orders of magnitude larger than that of an electron; hence, protons are the predominant carriers of mass, momentum, and energy of the solar wind.

The deflection of solar wind by the magnetopause forces the protons to transfer fractional bulk flow energy to thermal energy, as well as to magnetic energy. More importantly, some bulk flow energy of the protons even gets transferred to thermal energy of the electrons via a complicated wave particle interaction. The last statement is supported by the fact that because protons carry significantly more kinetic energy in the bulk flow than do the electrons, the $T_{\mathrm{i}} / T_{\mathrm{e}}$ ratio after the bow shock should rise to a high value (dozens); however, observations have shown that this value remains at the order of 5-12 (Paschmann et al., 1993; Phan et al., 1994). The underlying physics behind this energy transfer from protons to electrons could be that the more thermally active electrons have a greater mobility to satisfy the electric neutrality.

For further entry of solar-originated particles into the magneto- sphere, the $T_{\mathrm{i}} / T_{\mathrm{e}}$ ratio would face two tendencies. First, when the magnetosheath particles enter the magnetosphere, the $T_{\mathrm{i}} / T_{\mathrm{e}}$ ratio would increase because the energy in the bulk flow is further transferred to thermal energy (the magnetosphere is a rest reference frame relative to the solar wind). Second, the $T_{\mathrm{i}} / T_{\mathrm{e}}$ ratio would decrease if particles receive much more energy than their magnetosheath values because in the magnetospheric reference frame, the equilibrium state requires an energy balance between protons and electrons.

Under a southward IMF condition, it is commonly acknowledged that the magnetic reconnections on the dayside magnetopause and in the night-side magnetotail play dominant roles in loading the solar wind plasma into the plasma sheet.

Under a northward IMF condition, this situation is considerably subtle. The entry process is unclear and this issue is currently an active area of research.

The entry processes of the magnetosheath particles into the magnetosphere may be divided into three categories. Here, the magnetosphere refers to the part of the magnetosphere, where both ends of a magnetic field line are connected to the Earth (forming a closed magnetic flux tube), and the entry refers to the particles crossing the magnetopause. The first category is a direct embedding of a portion of a magnetosheath magnetic flux tube into the magnetosphere. The second category is the magnetosheathcharged particle crossing the magnetic field line from the magnetosheath to the magnetosphere. Finally, the third category is the conjugate (related to the equator) reconnections of the magnetosheath magnetic flux tube with the closed magnetic flux tube of the magnetosphere, thus replacing a portion of the closed magnetic flux tube of the magnetosphere with a portion of the magnetosheath magnetic flux tube.

The current prevailing mechanisms describing the entry of the solar plasma into the plasma sheet under a northward IMF are double-cusp or double-lobe reconnection (the third category), the Kelvin-Helmholtz instability (the third category), kinetic Alfvén waves (the second category), and impulsive penetration (the first category) (Wing et al., 2014). Although these mechanisms can explain the observed plasma density in the plasma sheet, no theoretical studies on the observed $T_{\mathrm{i}} / T_{\mathrm{e}}$ ratio have been conducted.

In addition, the $T_{\mathrm{i}} / T_{\mathrm{e}}$ ratio, which is preserved (observationally) as the particles cross the magnetopause, we also use another parameter, namely the specific entropy, to narrow down the selection. The specific entropy is defined as follows.

$$
s=p / \rho^{\gamma},
$$

where $p$ is the thermal pressure; $\rho$ is the density; and $\gamma$ is the polytropic index. For an adiabatic process, the specific entropy is preserved. However, observations (Johnson and Wing, 2009) have shown that the specific entropy increases by two to three orders of magnitude, accompanying the crossing of the magnetopause. The parameter $s$ (an intensive quantity) is closely related to the total entropy (an extensive quantity). Here, the total entropy is defined as

$$
S=p V^{\gamma}
$$


where $v$ is the volume of the unit magnetic flux tube. Substituting density with mass per unit volume in Equation (1), we obtain

$$
s=p /(M / V)^{\gamma}=p V^{\gamma} / M^{\gamma}=S / M^{\gamma},
$$

where $M$ is the mass contained in the volume of the unit magnetic flux tube. Thus, for an adiabatic process, the total entropy is preserved, which for ions is

$$
S_{\mathrm{i}}=p_{\mathrm{i}} V^{\gamma}
$$

where subscript i represents the ions. Substituting $p_{\mathrm{i}}=n k T_{\mathrm{i}}$ (where $n$ is the number density of ions and $k$ is Boltzmann's constant) into Equation (4), we obtain

$$
S_{\mathrm{i}}=n k T_{\mathrm{i}} V^{\gamma}
$$

The total entropy of the electrons is

$$
S_{\mathrm{e}}=p_{\mathrm{e}} V^{\gamma},
$$

where subscript e represents the electrons. Substituting $p_{\mathrm{e}}=n k T_{\mathrm{e}}$ into Equation (6), we obtain:

$$
S_{\mathrm{e}}=n k T_{\mathrm{e}} V^{\gamma}
$$

From Equations (5) and (7), it is clear that for an adiabatic process, the preservation of the total entropies of both ions and electrons leads to the preservation of the $T_{\mathrm{i}} / T_{\mathrm{e}}$ ratio. It should be noted that the reverse may not be true; that is, the preservation of the $T_{\mathrm{i}} / T_{\mathrm{e}}$ ratio does not imply an adiabatic process.

The preservation of the $T_{\mathrm{i}} / T_{\mathrm{e}}$ ratio and the increment of the specific entropy by two to three orders of magnitude accompanying the crossing of the magnetopause impose two very strong constraints on the candidate entry process. The qualified process should be a non-adiabatic process, which preserves the $T_{\mathrm{i}} / T_{\mathrm{e}}$ ratio. In addition, this process should accelerate the particles dramatically, which in turn, significantly heats the cool dense magnetosheath particles to hot tenuous magnetospheric particles.

Now, let us examine the entry processes of the plasma one by one. Firstly, we consider the impulsive penetration (Echim and Lemaire, 2000). An impulsive entry of magnetosheath plasma blobs can occur if the magnetosheath irregularities have sufficient momentum to cross the magnetopause. At this moment, both ends of the embedded flux tube are still connected with the IMF, and not with the Earth. The magnetic moment is conserved, which is defined as

$$
\mu=\frac{m v_{\perp}^{2} / 2}{B}
$$

where $m$ is the mass of a particle; $v_{\perp}$ is the thermal speed of the particle perpendicular to the magnetic field; and $B$ is the magnetic field. Equation (8) can be rewritten as follows:

$$
\mu=\frac{m(\boldsymbol{V}-\boldsymbol{u})_{\perp}^{2} / 2}{\boldsymbol{B}}
$$

where $\boldsymbol{V}$ is the velocity of a particle and $\boldsymbol{u}$ is the bulk velocity of the penetrating irregular magnetosheath plasma blob; then, the process can be easily understood. From Equation (9), the conservation of the magnetic moment of the electrons and ions requires the reduction of $u$, as the magnetosheath plasma blob penetrates deep into the magnetosphere with an increasing magnetic field $\boldsymbol{B}$.
The irregularities are slowed down adiabatically as a consequence of the conservation of the magnetic moments of the electrons and ions. Expressing the conservation of the magnetic moment explicitly, for the ions we obtain

$$
\mu_{\mathrm{i} \text {,sheath }}=\frac{m_{\mathrm{i}} v_{\mathrm{i}, \perp, \text { sheath }}^{2} / 2}{B_{\text {sheath }}}=\frac{k T_{\mathrm{i}, \perp \text {,sheath }}}{B_{\text {sheath }}}=\mu_{\mathrm{i}, \text { sphere }}=\frac{k T_{\mathrm{i}, \perp \text {,sphere }}}{B_{\text {sphere }}},
$$

and for the electrons,

$$
\mu_{\mathrm{e}, \text { sheath }}=\frac{m_{\mathrm{e}} v_{\mathrm{e}, \perp, \text { sheath }}^{2} / 2}{B_{\text {sheath }}}=\frac{k T_{\mathrm{e}, \perp, \text { sheath }}}{B_{\text {sheath }}}=\mu_{\mathrm{e}, \text { sphere }}=\frac{k T_{\mathrm{e}, \perp, \text { sphere }}}{B_{\text {sphere }}} .
$$

Dividing Equation (10) by Equation (11), we obtain

$$
\frac{T_{\mathrm{i}, \perp, \text { sheath }}}{T_{\mathrm{e}, \perp, \text { sheath }}}=\frac{T_{\mathrm{i}, \perp, \text { sphere }}}{T_{\mathrm{e}, \perp, \text { sphere }}} \text {. }
$$

This mechanism has the advantage of preserving the ion-to-electron perpendicular temperature ratio as the ions and electrons pass through the magnetopause. Although the ion-to-electron perpendicular temperature ratio is preserved, the adiabatic characteristic of the impulsive penetration is not consistent with the increment of the specific entropy, which is a non-adiabatic requirement of the candidate process.

Secondly, we consider kinetic Alfvén waves (Hasegawa and Mima, 1978; Johnson and Cheng, 1997; Izutsu et al., 2012). Larger amplitude Alfvén waves can excite the ions to cross the magnetic field lines as diffusion. This process can heat the plasma to the magnetospheric temperature. The diffusion process is determined by the following equation:

$$
\frac{\partial n_{i}}{\partial t}=\frac{\partial}{\partial x} D_{i, \perp} \frac{\partial n_{i}}{\partial x}
$$

where $n_{\mathrm{i}}$ is the number density of ions and $D_{\mathrm{i}, \perp}$ is the diffusion coefficient of ions, which in the kinetic Alfvén waves, is proportional to the energy as

$$
D_{\mathrm{i}, \perp} \propto T_{\mathrm{i}}
$$

However, the diffusion coefficient is energy-dependent, but not mass dependent. Electric neutrality requires that electrons catch up with the protons. Equation (14) indicates that they will need to obtain an equal amount of energy $\left(T_{\mathrm{e}} \approx T_{\mathrm{i}}\right)$ to do so. This may violate the preservation of the $T_{\mathrm{i}} / T_{\mathrm{e}}$ ratio. Even the electrons coming from the ionosphere cannot guarantee the preservation of the ratio.

Thirdly, we consider the double-cusp or double-lobe reconnection (Song P and Russell, 1992). This process replaces the portion of the closed magnetic flux tube of the magnetosphere beyond the northern and southern cusps with a portion of the magnetosheath magnetic flux tube. The distance between the conjugate northern and southern reconnection sites can be estimated to be of the order of $20 R_{e}$; the duration of the reconnection is estimated to be of the order of one min (Onsager et al., 2001); and the accelerated electron energy is of the order of $20 \mathrm{eV}$ (Lavraud et al., 2006). From the appendix, we know that this mechanism has a great probability of preserving the $T_{\mathrm{i}} / T_{\mathrm{e}}$ ratio because the traveling time (50 s) of the energized electrons between the conjugated reconnection sites is approximately the same as the duration 
of the reconnection. Because of the convergence of the magnetic field lines toward the cusps and high latitude of the reconnection sites, a major portion of the original magnetospheric flux tube is replaced by the magnetosheath magnetic flux tube, and the particles contained inside the post-reconnected flux tube are mostly magntosheath-originated. From Equation (2), it is clear that the conjugate reconnections at the cusp or lobe do not significantly increase the total entropy (Johnson and Wing, 2009). Thus, from Equation (3), the specific entropy of the plasma does not increase to the magnetospheric value across the magnetopause. The double-cusp reconnection plays the role of replacing the magnetospheric flux tube with the magnetosheath flux tube and the particles within.

Finally, we turn to the Kelvin-Helmholtz instability (Otto and Fairfield, 2000; Nykyri et al., 2006; Li W et al., 2016). Contrary to the double-cusp reconnections, which take place at high latitudes, the conjugate reconnections in the nonlinear phase of the instability occur at low latitudes, and replace the portion of the magnetospheric flux tube near the equator with a portion of the magnetosheath flux tube. Only a minor volume of the original magnetospheric flux tube is replaced. Because of the convergence of the magnetic field lines toward both northern and southern cusps, a closed magnetospheric magnetic flux tube has significantly larger cross section near the equator than that at the cusp, and the conjugate reconnection sites near the equator have significantly longer " $X$ " line extension than those at the cusps. Consequently, more particles are involved in the reconnection in the nonlinear phase of the Kelvin-Helmholtz instability than those in the double-cusp or double-lobe reconnection. The conjugate reconnections in the nonlinear phase of the instability have the potential to increase the specific entropy of the plasma to the magnetospheric level. The distance between the conjugate northern and southern reconnection sites can be estimated to be of the order of $4 R_{\mathrm{e}}$; the duration of the reconnection is estimated to be of the order of $10 \mathrm{~s}$ (Faganello et al., 2012); and the accelerated electron energy is of the order of $20 \mathrm{eV}$ (Nykyri et al., 2006). From the appendix, we know that this mechanism has a large probability of preserving the $T_{\mathrm{i}} / T_{\mathrm{e}}$ ratio because the traveling time (9.6 s) of the energized electrons between the conjugated reconnection sites is of the same order as the duration of the reconnection.

From the above analysis, we arrive at the following understanding: The impulsive penetration would satisfy the first constraint of the preservation of the $T_{\mathrm{i}} / T_{\mathrm{e}}$ ratio, but not the second constraint of the increment of the specific entropy. The kinetic Alfvén waves could not satisfy the first constraint of the preservation of the $T_{\mathrm{i}} / T_{\mathrm{e}}$ ratio; however, it has the potential to raise the specific entropy of the plasma to the magnetospheric value of the second constraint. The double-cusp or double-lobe reconnection has the potential to preserve the $T_{\mathrm{i}} / T_{\mathrm{e}}$ ratio of the first constraint; however, it does not have the ability to raise the specific entropy to the magnetospheric level of the second constraint. Only the Kelvin-Helmholtz instability has the potential to satisfy both the constraints of the preservation of the temperature ratio and the increment of the specific entropy.

Based on the above discussion, we chose the Kelvin-Helmholtz instability as the dominant entry mechanism of solar-originated plasma, followed by the double-cusp or double-lobe reconnection, kinetic Alfvén waves, and impulsive penetration.

\section{Transport of Charged Particles within the Plasma Sheet}

The factors that may affect the $T_{\mathrm{i}} / T_{\mathrm{e}}$ ratio in the plasma sheet include the ionosphere, patchy tail reconnection, and bursty bulk flows (BBFs).

The ionosphere could serve as an energy sink for the magnetospheric particles. For the study of the $T_{\mathrm{i}} / T_{\mathrm{e}}$ ratio, which requires the exchange of fractional particles between the magnetosphere and ionosphere in a short time (in approximately $20 \mathrm{~min}$ ), this effect is negligible because the number flux of the precipitation particles is too small to accomplish this task. For example, taking the precipitation number flux as $7.93 \times 10^{6} \mathrm{~cm}^{-2} \mathrm{sec}^{-1} \mathrm{sr}^{-1}$ (Hardy et al., 1989), a flux tube extending to $20 R_{\mathrm{e}}$ down tail with a number density of $0.3 \mathrm{~cm}^{-3}$ will take about $140 \mathrm{~h}$ to be replaced. Thus, the ionosphere should be treated only as a reflector of the magnetospheric particles in the present investigation.

To investigate the effect of patchy tail reconnection on the $T_{\mathrm{i}} / T_{\mathrm{e}}$ ratio in the plasma sheet, we apply Equation (A32) for the magnetospheric model. The Tsyganenko (96) magnetospheric magnetic field model (Tsyganenko, 1995) and Tsyganenko tail plasma sheet model (Tsyganenko and Mukai, 2003) are used for the magnetic field and plasma, respectively, where the input parameters are: a solar wind density of $6 \mathrm{~cm}^{-3}$; velocity of $380 \mathrm{~km} / \mathrm{s}$; IMF $B_{z}=-5 \mathrm{nT}$, and Dst $=-80 \mathrm{nT}$.
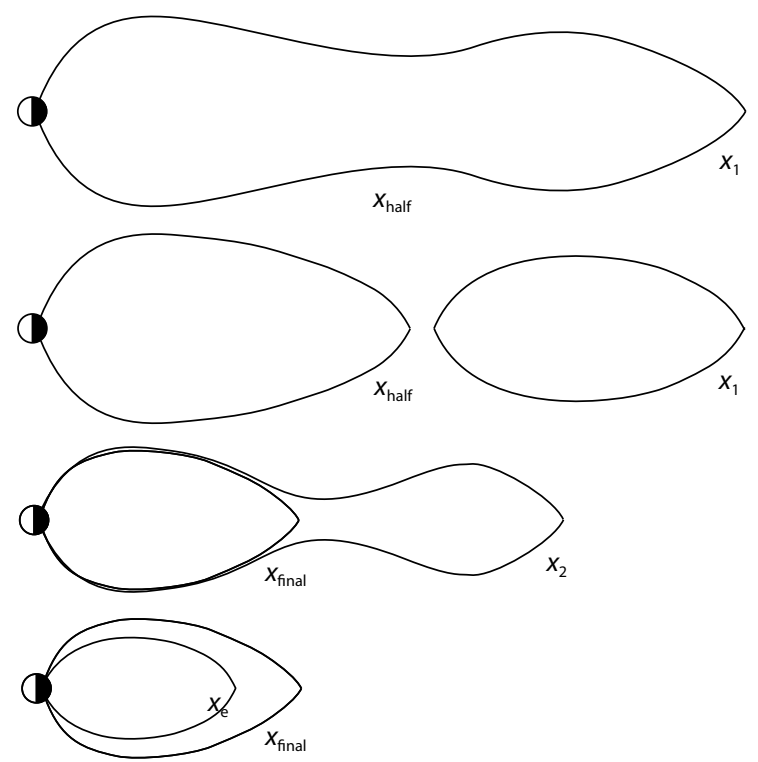

Figure 1. Schematic diagram showing patchy magnetic reconnections and the related positions. A flux tube, whose $T_{\mathrm{i}} / T_{\mathrm{e}}$ ratio is under investigation and is originally extending to $x_{1}\left(x_{1}=2 x_{\text {half }}\right)$, is cut by a reconnection taking place at $x_{\text {half }}$, and has its final position at $x_{\text {final. }}$ A series of neighboring background medium flux tubes (with their $y$ values slightly different from those of the one under investigation), originally extending between $x_{2}\left(x_{2}=2 x_{\text {final }}\right)$ and $x_{1}$, are cut by a series of reconnections taking place between $x_{\text {final }}$ and $x_{\text {half, }}$, and have their final positions between $x_{\mathrm{e}}$ and $x_{\text {final }}$. 
Figure 1 schematically illustrates the procedure followed in our calculation. From the magnetic field model, the equatorial point $x_{1}$ is first located. Then, the magnetic field line passing through this point is traced, and the length $2 L$ of this field line from the equatorial point to the ionosphere is recorded. The plasma data on this field line are determined using the tail plasma sheet model.

Assume that a patchy magnetic reconnection occurs at the midpoint $x_{\text {half }}$ of this field line (Borovsky et al., 1998). The cut flux tube moves earthward as a BBF. The total entropy of the earthward half of the cut flux tube is calculated and compared with the entropies of the equatorial plane points with the same $y$ value. The earthward motion would stop at a location where the total entropy of the background plasma is the same as the entropy of the cut flux tube. The $x$ value of the matched point would be the final position $x_{\text {final }}$ of the post-reconnected flux tube (Chen CX and Wolf, 1999).

Figure 2 shows a representative calculation. For the reconnection, the electric field $E$ was set to $5 \mathrm{mV} / \mathrm{m}$; the time duration of the reconnection $\Delta t$ was set to $60 \mathrm{~s}$; and the $z$-directional magnetic field $B_{z}$ was set to $1.0 \mathrm{nT}$. As can be seen from the equations in the appendix, these quantities are not independent of each other. We also experimented with other values of $E$ from 4 to $10 \mathrm{mV} / \mathrm{m}, \Delta t$ from 40 to $80 \mathrm{~s}$, and $B_{z}$ from 0.5 to $1.0 \mathrm{nT}$, and the results are similar.

The domain of our calculation $\left(x: 10-80 R_{\mathrm{e}}, y:-20\right.$ to $20 R_{\mathrm{e}}$ ) is limited by the domain of the Tsyganenko tail plasma sheet model, which is valid for $10-50 R_{\mathrm{e}}$ down the Earth's tail. For simplicity, we linearly interpolated the equatorial plasma data between $50 R_{e}$ and $220 R_{\mathrm{e}}$ down the tail with a plasma number density of $0.3 \mathrm{~cm}^{-3}$ and an electron temperature of $1.2 \times 10^{6} \mathrm{~K}$ at $220 R_{\mathrm{e}}$ (Slavin et al., 1985).

The magnetic field strength at the midpoint of each pre-reconnected field line is represented as $\boldsymbol{B}$ in the calculation. The electron temperature was set to be one-sixth of the ion temperature (i.e., close to the ratio in the magnetosheath (Wang CP et al., 2012)) in each pre-reconnected field line.
The calculated $T_{\mathrm{i}} / T_{\mathrm{e}}$ ratio in the equatorial plane earthward of $60 R_{\mathrm{e}}$ is comparable to that reported by Chen CX and Wang CP (2019). In particular, the closer the position is to the Earth, the lower was the ratio. Furthermore, the ratio is slightly higher at dawn or dusk than at midnight. It is noteworthy that the $T_{\mathrm{i}} / T_{\mathrm{e}}$ ratio is preserved tailward of $60 R_{\mathrm{e}}$ because of the large distance between the reconnection site and the ionosphere.

BBFs may affect the $T_{\mathrm{i}} / T_{\mathrm{e}}$ ratio through the turbulence on their boundaries. Following the treatment of Chen CX (2013), we consider the viscosity as

$$
\mu=\frac{1}{3}\left(\rho \bar{U}_{\mathrm{i}} \bar{\lambda}\right)
$$

where $\rho$ is the mass density; $\bar{U}_{\mathrm{i}}$ is the mean thermal speed defined by Equation (A15); and $\bar{\lambda}$ is the mean free path length, which in the collisionless Earth's magnetosphere can be taken as

$$
\bar{\lambda}=2 a_{\mathrm{i}} \text {. }
$$

The gyroradius $a_{i}$ is defined by Equation (A1). As the ions possess a significantly larger gyroradius than do the electrons, the contribution to viscosity is mainly from the ions.

In a collision-less environment, the mean free path is meaningful only when wave-particle interactions are involved. In the presence of a magnetic field, the farthest distance a charged particle can move in a cross-magnetic field direction is twice that of its gyroradius.

Assume that a newly cut flux tube moves from $x_{\text {half }}$ to $x_{\text {final, }}$ and the motion in the zeroth-order approximation is adiabatic. Then, the relation can be written as

$$
p_{\text {final }} v_{\text {final }}^{5 / 3}=p_{\text {half }} v_{\text {half }}^{5 / 3}
$$

where $v$ is the upper half volume of the flux tube above the equatorial plane with a unit magnetic flux. Equation (17) states the conservation of total entropy.

Although there are two flanks of the fast flow exposed to the surrounding medium, only one half of the friction-producing heat flux enters the flux tube of the fast flow because the flanks are

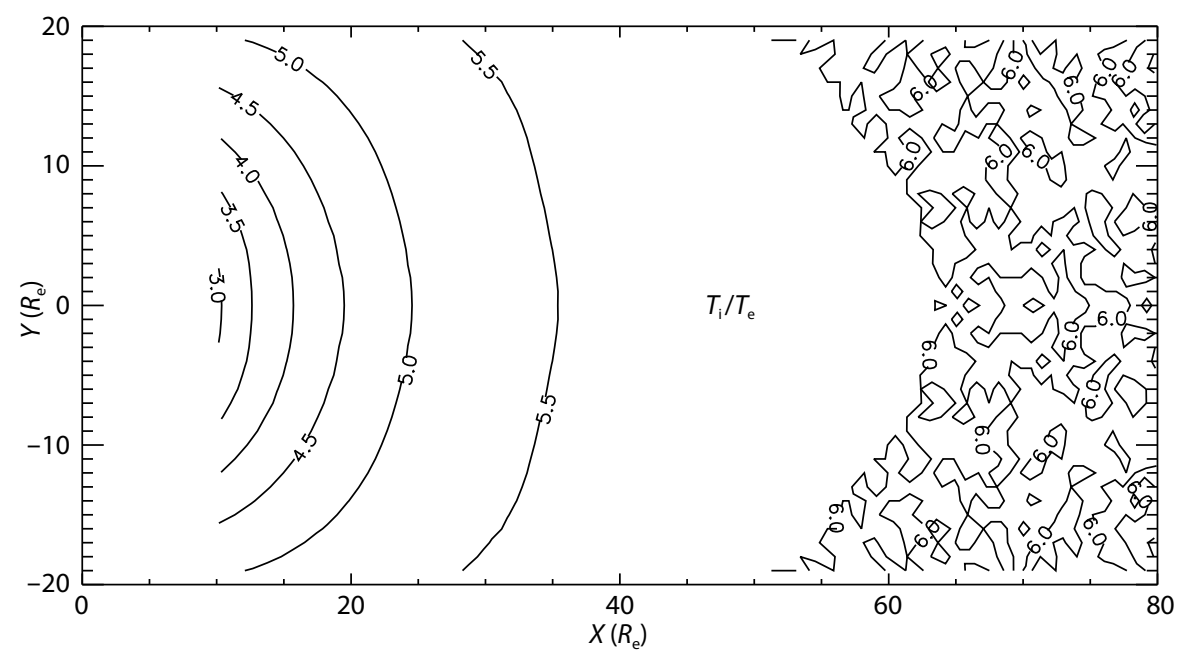

Figure 2. Contours of the calculated $T_{\mathrm{i}} / T_{\mathrm{e}}$ ratio on the equatorial plane with only the influence of the electric field being considered. 
hotter than either the medium or the fast flow (scattering of ions by turbulent electric field). For simplicity, the heat flux is assumed to be split between the medium and the flux tube of the fast flow. Because the turbulent electric fields (induced by the cross magnetic field motion of flow) basically transfer the plasma bulk flow energy to thermal energy, and our expression for particle energy (from a microscopic point of view) does not distinguish between the bulk flow energy (a macroscopic quantity) and thermal energy, the electron energy does not change within the flux tube. Thus, the role of electrons in the turbulence is negligible. In contrast, because of their much larger gyroradii (easily crossing the boundaries of the BBFs), the ions receive and/or give energy to the surrounding medium through the turbulent electric fields. Thus, the heat flux is only distributed among the ions, as only the ions scattered by the turbulent electric field, are the carriers of the heat flux, and the energy exchange between ions and electrons is inefficient (otherwise, the $T_{\mathrm{i}} / T_{\mathrm{e}}$ ratio would approach one).

The friction-producing heat flux affects the newly reconnected flux tube in two ways. The first is the heat that this flux tube receives from the fast flows passing by (in this step of calculation, the flux tube we are concerned with is treated as a medium), which can be expressed as

$$
W_{\text {frii }}=\mu \frac{\partial u}{\partial y}\left(x_{e}-x_{\text {final }}\right) D_{z} D_{x}
$$

where $u$ is the speed of fast flow; $y$ is pointed in the cross tail direction; $x_{\mathrm{e}}$ (shown in Figure 1 ) is the earthward stop position of a passing by fast flow (a flux tube that originally extends to $x_{2}$ ) formed (reconnected) at $x_{\text {final }} ; D_{z}$ is the $z$-directional effective dimension for friction; and $D_{x}$ is the $x$-directional dimension of fast flow. It is noteworthy that Equation (18) accounts for the contribution from a series of fast flows, whose final positions occupy points between $x_{\mathrm{e}}$ and $x_{\text {final }}$.

Substituting $\rho=n m_{\mathrm{i}}$, and Equations (15) and (16) into Equation (18), we obtain

$$
W_{\text {fri1 }}=\frac{1}{3} n_{\text {final }} m_{\mathrm{i}} \frac{16 K T_{\mathrm{i}, \text { final }}}{\pi e B_{\text {final }}} \frac{U}{D_{y}}\left(x_{\mathrm{e}}-x_{\text {final }}\right) D_{z} D_{x \prime}
$$

where $U$ is the typical speed of the BBFs, which is approximately $400 \mathrm{~km} / \mathrm{s}$ and $D_{y}$ is the length scale of the variation of the fast flow in $y$-direction.

The second is the heat that a flux tube transfers to the background medium at a distance from $x_{\text {half }}$ to $x_{\text {finall }}$, which can be expressed as

$$
W_{\text {fri2 }}=\frac{1}{3} n_{\text {fh }} m_{\mathrm{i}} \frac{16 K T_{\mathrm{i}, \mathrm{fh}}}{\pi e B_{\mathrm{fh}}} \frac{U}{D_{y}}\left(x_{\text {final }}-x_{\text {half }}\right) D_{z} D_{x}
$$

where subscript fh represents the middle point between $x_{\text {half }}$ and $x_{\text {final }}$.

Three points are critical here: (i) point $x_{\text {final, }}$ at which we are currently interested in the ratio; (ii) point $x_{\text {half }}$, where the flux tube of our interest comes from; and (iii) point $x_{\mathrm{e}}$, which is the earthward position that a newly reconnected (at point $x_{\text {final }}$ ) flux tube can reach. A flow with its stop position earthward of $x_{\mathrm{e}}$ would be generated earthward of $x_{\text {final }}$ and thus, would not contribute any heat to point $x_{\text {finali }}$ similarly, the flux tube whose $T_{\mathrm{i}} / T_{\mathrm{e}}$ ratio we are inter- ested in, would not distribute any heat to points tailward of $x_{\text {half }}$ because our flux tube was created earthward of those points.

We assume that $D_{y}$ is of the order of the cross tail width of the BBFs $\left(D_{y}=3 R_{\mathrm{e}}\right)$. The equatorial cross-section of the fast flow is set to $3 R_{\mathrm{e}} \times 3 R_{\mathrm{e}} ; D_{\mathrm{x}}$ is set to $3 R_{\mathrm{e}}$; and $D_{z}$ is set to $2 R_{\mathrm{e}}$.

The $T_{\mathrm{i}} / T_{\mathrm{e}}$ ratio at $x_{\text {final }}$ can be estimated as

$$
\frac{T_{\mathrm{i}, \text { final }}}{T_{\mathrm{e}, \text { final }}}=\frac{\frac{3}{2} p_{\text {i,final }} V_{\text {final }}+W_{\text {fri } 1}-W_{\text {fri2 }}}{\frac{3}{2} p_{\text {e,final }} V_{\text {final }}},
$$

Substituting Equation (17) into Equation (21), we obtain

$$
\frac{T_{\text {i,final }}}{T_{\text {e,final }}}=\frac{\frac{3}{2} p_{\text {i, half }} V_{\text {half }}\left(V_{\text {half }} / V_{\text {final }}\right)^{2 / 3}+W_{\text {fri1 }}-W_{\text {fri2 }}}{\frac{3}{2} p_{\text {e, half }} V_{\text {half }}\left(V_{\text {half }} / V_{\text {final }}\right)^{2 / 3}}
$$

Substituting $p=n k T$, and Equations (A21, A22, A24, and A28) into Equation (22), we obtain:

$$
\frac{T_{\mathrm{i}, \text { final }}}{T_{\mathrm{e}, \text { final }}}=\frac{\left(W_{\mathrm{i}}+\Delta W_{\mathrm{i}}\right) D_{y} D_{x} B_{\text {half }}\left(V_{\text {half }} / V_{\text {final }}\right)^{2 / 3}+W_{\text {fri1 }}-W_{\text {fri2 } 2}}{\left(W_{\mathrm{e}}+\Delta \mathrm{W}_{\mathrm{e}}\right) D_{y} D_{x} B_{\text {half }}\left(V_{\text {half }} / V_{\text {final }}\right)^{2 / 3}},
$$

where $W_{\mathrm{i}}+\Delta W_{\mathrm{i}}$ and $W_{\mathrm{e}}+\Delta W_{\mathrm{e}}$ are estimated in a volume of one unit magnetic flux.

Figure 3 shows the influence of turbulence alone. It can be seen that earthward of $60 R_{e}$, turbulence has an insignificant influence on the $T_{\mathrm{i}} / T_{\mathrm{e}}$ ratio. The reason for this can be seen from Equation (23), wherein the ratio of the heat induced by friction to the total energy of the particles is an inverse function of the magnetic field strength on the equatorial plane. The closer a position is to the Earth, the less the turbulence contributes to the total energy.

Figure 4 demonstrates the distribution of the $T_{\mathrm{i}} / T_{\mathrm{e}}$ ratio with all the influencing factors included. The tendency of the increase of the ratio from far tail to midtail is consistent with the observation by Slavin et al. (1985).

It is interesting to notice that there is a plateau in the down tail distance over $40-60 R_{\mathrm{e}}$. This may explain the high correlation between the ion and electron temperatures in the outer central plasma sheet, as reported by Baumjohann et al. (1989). The patchy reconnection and turbulence may counteract each other in this region.

When the IMF is northward, rare magnetic reconnections occur inside the plasma sheet. In this case, the $T_{\mathrm{i}} / T_{\mathrm{e}}$ ratio will be preserved. This conclusion is consistent with the observed cool plasma distribution in the third panel of Fig. 6(b, c) of Wang CP et al. (2012).

\section{Discussion}

Prima facie, it appears that all the physical processes in the magnetosphere are involved in the variation or preservation of the $T_{\mathrm{i}} / T_{\mathrm{e}}$ ratio. However, the number of dominant processes that can affect the temperature ratio is very limited because the candidate processes should be "powerful enough". The observation-based statistical $T_{\mathrm{i}} / T_{\mathrm{e}}$ ratio is a function of the geomagnetic activity level. The time scale of the variation of the observational temperature ratio is of the order of a few tens of min (for example, of the order 


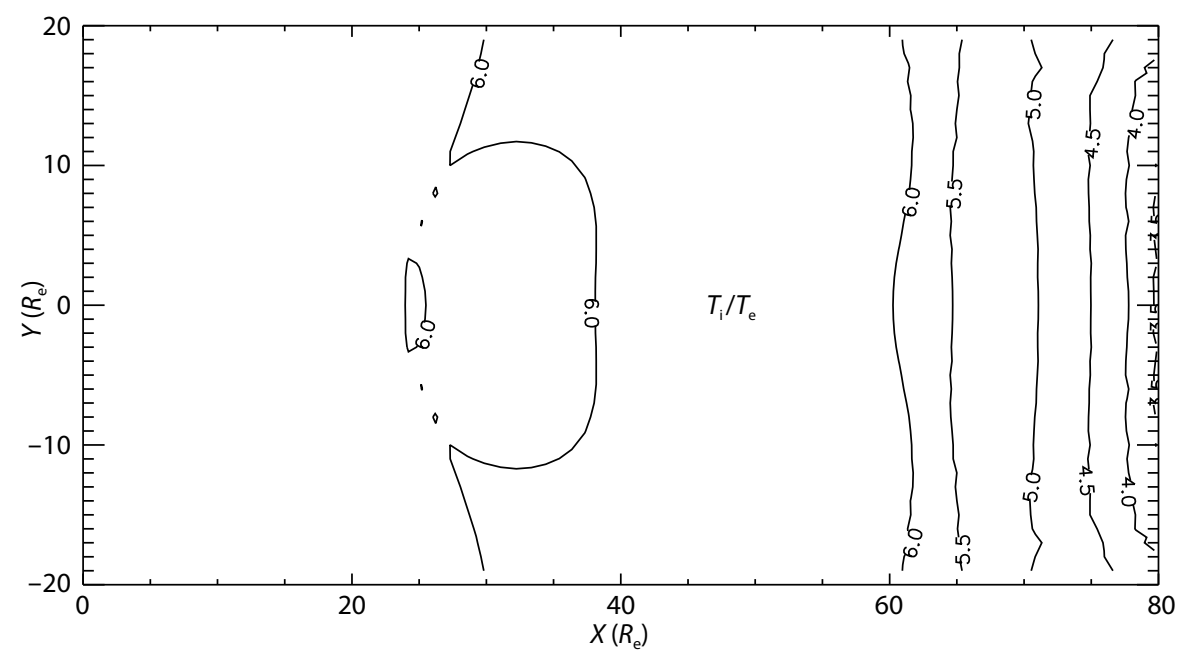

Figure 3. Contours of the calculated $T_{\mathrm{i}} / T_{\mathrm{e}}$ ratio on the equatorial plane with only the influence of turbulence being considered.

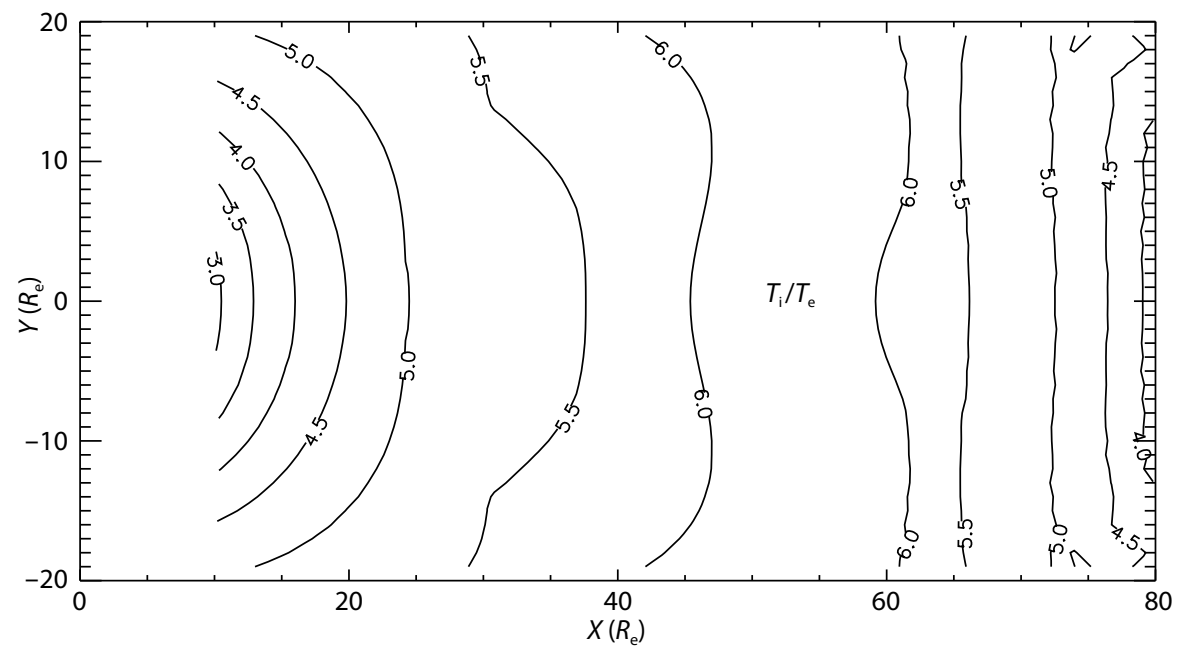

Figure 4. Contours of the calculated $T_{\mathrm{i}} / T_{\mathrm{e}}$ ratio on the equatorial plane with influences of both the electric field and turbulence being considered.

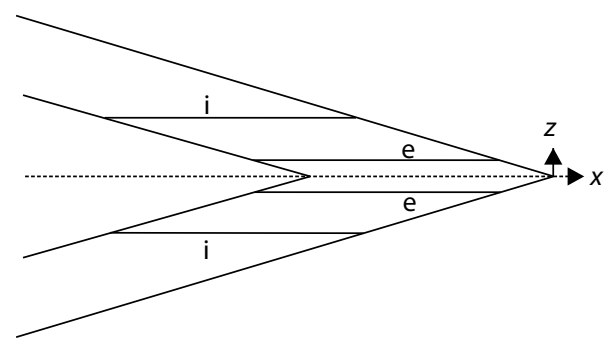

Figure 5. Schematic diagram depicting the left part of the X-line. The oblique magnetic field lines are in the $x z$-plane and the $x$-axis indicates the neutral sheet. The area between the line labeled " $\mathrm{e}$ " and the neutral sheet represents the non-adiabatic motion domain for the electrons, and the area between the line labeled " $\mathrm{i}$ " and the neutral sheet represents the non-adiabatic motion domain for the ions.

of the duration of the BBFs). By the term "powerful enough", we imply that a process should have power to change, on an average, a significant fraction of the energy of every particle inside a flux tube in a short time which is comparable to the time scale of the variation of the observational temperature ratio. Thus, many processes can be ignored based on this criterion, and only some powerful processes qualify on this basis. Consequently, an approximation can be made to keep only the lowest order as long as a characteristic of the preservation or variation of the temperature ratio can be demonstrated.

Our study demonstrated that patchy magnetic reconnection plays a role in the preservation or variation of the $T_{\mathrm{i}} / T_{\mathrm{e}}$ ratio during solar wind plasma entry into the plasma sheet under both southward and northward IMF conditions.

A significant difference between the present study and those of Onsager et al. (1991) and Schriver et al. (1998) is our consideration of the electric field, experienced by the charged particles in the plasma sheet. In our study, the electric field is not assumed to be constant and steady, but is enhanced in some local channels (with varying lengths from the Earth) and short-lived. (there exist some electric fields, which are imposed on both the magnetic reconnection site and resulting BBF (with a typical width of $2-3 R_{\mathrm{e}}$ ). In our calculation, the electric field is applied to the reconnection 
site explicitly, as described in the sixth paragraph of Section 3. The electric field applied to the BBF is given only implicitly because the earthward motion of the BBF implies that there is an electric field inside the flow channel.) In addition, there are turbulent electric fields on the boundaries of these channels, which are also given implicitly through an effective friction. The acceleration of the charged particles in such a spatially and temporally short and turbulent electric field is fundamentally different from that under a uniform and steady electric field.

The location of a reconnection site, or more precisely, the distance from a reconnection site to the magnetic mirror point, plays a crucial role in the preservation or variation of the $T_{\mathrm{i}} / T_{\mathrm{e}}$ ratio. The non-preservation of this ratio comes from terms higher than the second one on the right-hand side of Equations (A24) and (A28). In other words, this ratio is preserved, when the effective time duration of reconnection is less than the sum of the half-cyclotron period and round-trip travel time from the reconnection site to the magnetic mirror point for both the accelerated ions and electrons. Because the mass of an electron is significantly less than that of an ion, the cyclotron period is correspondingly less for the electrons than it is for the ions, as can be seen from Equations (A4) and (A8). For the observed $T_{\mathrm{i}} / T_{\mathrm{e}}$ ratio around the magnetosphere, the electrons have a much higher thermal speed than the ions. Consequently, the round-trip travel time from the reconnection site to the mirror point and back is significantly less for electrons than it is for the ions, as can be seen from Equations (A25) and (A29). In the region earthward of $60 R_{e}$, the ions experience nonadiabatic acceleration no more than once, while most electrons experience non-adiabatic acceleration more than once, which leads to a lower ratio than that of the magnetosheath.

In our calculation, note that the convection is accomplished mainly (60\%-100\%) by BBFs (Angelopoulos et al., 1994), and BBFs are produced by magnetic reconnections. In this simplified picture, for plasma to be transported from the far tail (approximately $100 R_{\mathrm{e}}$ and beyond) to the near-Earth tail (approximately $6-10 R_{\mathrm{e}}$ ), more than one magnetic reconnections on the same flux tube are required. For example, a flux tube extending to $(208.0,0.0)$ is cut by a reconnection at point $(102.9,0.0)$, approaching its final position $(79.9,0.0)$. In another example, a flux tube extending to (48.0, $0.0)$ is cut by a reconnection at point $(24.0,0.0)$, with a final position of $(10.2,0.0)$. Only when the total entropy (defined by Equation (2)) of the flux tube is reduced, is it possible for the cut flux tube to move earthward.

Globally, the decrease in the $T_{\mathrm{i}} / T_{\mathrm{e}}$ ratio down the far tail is a demonstration of the violation of frozen-in condition of the ions. Typically, ions with a larger gyroradius than electrons are more significantly affected by a turbulent electric field than the electrons. They would not stay in the same earthward fast moving flux tube to experience the same magnetic compression as electrons, resulting in a decrease in the $T_{\mathrm{i}} / T_{\mathrm{e}}$ ratio.

The contours of the $T_{\mathrm{i}} / T_{\mathrm{e}}$ ratio on the equatorial plane in our calculation are closely related to the southward IMF condition. For the northward IMF, the situation is subtle because there are only a few reconnections and probably a few fast flows in the plasma sheet. In such a case, the path of charged particles from the flanks of the magnetosphere to the midnight remains uncertain. One possible mechanism proposed is eddy diffusion from the flank to the midnight (Wang CP et al., 2010). In this study, we have considered another mechanism, whereby the plasma would probably be dragged tailward along the flanks over some distance by the neighboring flow in the magnetosheath. In this tailward movement, the $T_{\mathrm{i}} / T_{\mathrm{e}}$ ratio is preserved, and specific entropy is increased. Panel 3 of Fig. 3e of Wang CP et al. (2012) may support this point of view because the decrease in the specific entropy of the ions indicates the energy lost from the flow just outside the magnetopause. After the magnetic tension is built, the flux tube turns into midnight and subsequently moves earthward (this scenario is similar to that proposed by Song $P$ et al. (1999)). In the turning and earthward motion, both the $T_{\mathrm{i}} / T_{\mathrm{e}}$ ratio and specific entropy are preserved because they are basically adiabatic processes.

The fact that the $T_{\mathrm{i}} / T_{\mathrm{e}}$ ratio of our calculation is not higher than that of the magnetosheath appears to support the view that the evolution of the warm plasma agrees with the second tendency of the general consideration in Section 2, while the $T_{\mathrm{i}} / T_{\mathrm{e}}$ ratio of the cooler plasma is preserved after the crossing.

It is noteworthy that the Speiser orbit is a special type of non-adiabatic trajectory in the current sheet. There are other types of nonadiabatic trajectories, such as cucumber and ring types (Büchner and Zelenyi, 1989). During the period of a patchy reconnection, the geometry of the magnetic field at the reconnection site would change dramatically from the normal condition; consequently, the distribution of charged particles among different trajectory types would also change. We think that in this period, the Speiser orbit is the dominant one.

\section{Summary}

In this study, we have applied equations derived for the energy gain of both the ions and electrons in a magnetic reconnection site (and/or on the boundaries of the resulting BBF) to the processes involved in solar-originated particles entering and being transported in the plasma sheet in geo-magnetotail. The conclusions drawn from this study are as follows:

(1) Patchy magnetic reconnection may preserve the $T_{\mathrm{i}} / T_{\mathrm{e}}$ ratio if charged particles are non-adiabatically accelerated no more than once in a single reconnection; on the other hand, multiple occurrences of non-adiabatic accelerations vary this ratio. The observed lower $T_{\mathrm{i}} / T_{\mathrm{e}}$ ratio near the Earth under a southward IMF confirms that the electrons reflected back from the magnetic mirror point experience multiple non-adiabatic accelerations.

(2) The Kelvin-Helmholtz instability is identified as the dominant process for solar-originated plasma entering the magnetosphere under a northward IMF. Consequently, the $T_{\mathrm{i}} / T_{\mathrm{e}}$ ratio is preserved in the plasma sheet, which is consistent with the results of the statistical investigation.

(3) The turbulence on the boundaries of BBF affects the $T_{\mathrm{i}} / T_{\mathrm{e}}$ ratio in the far tail through the violation of frozen-in condition of the ions. The calculated $T_{\mathrm{i}} / T_{\mathrm{e}}$ ratio decreases with the down-tail distance, which is consistent with the observations.

(4) The plateau in the contour of the estimated $T_{\mathrm{i}} / T_{\mathrm{e}}$ ratio in the 
down tail distance between 40 and $60 R_{\mathrm{e}}$ may explain the hitherto unresolved issue of the high correlation between the ion and electron temperatures in the outer central plasma sheet.

\section{Acknowledgments}

This work was supported by the National Nature Science Foundation of China (Grant NSFC41974204). Data were neither used nor created for this study.

\section{Appendix A}

\section{Influence of a Patchy Magnetic Reconnection on the Ion- to-Electron Temperature Ratio}

We will investigate the role of magnetic reconnection in the $T_{\mathrm{i}} / T_{\mathrm{e}}$ ratio. More precisely, we will investigate patchy magnetic reconnection (i.e., short time duration and short X-line length) in this context.

Figure 5 shows the left side of the X-line. In the following explanation, we will concentrate on the upper half of this picture in more detail.

The electric field is represented by $E$; the $x$-extension of the cross section of the flux tube is $\Delta x$; the X-line length is $\Delta y$ (Nakamura et al., 2004; Chen CX 2013); the time duration of reconnection is $\Delta t$; the number density is $n$; the magnetic field is $\boldsymbol{B}$; and the ion and electron temperatures in the pre-reconnected flux tube are $T_{i}$ and $T_{\mathrm{e}}$, respectively. If we assume that the $z$-extension of the domain of the non-adiabatic motion of the ions (i.e., the order of ion gyroradius in the magnetic field $B$ owing to the meandering motion around the neutral sheet in the yz-plane (Hill, 1975)) is

$$
a_{i}=\frac{m_{\mathrm{i}} \bar{U}_{\mathrm{i}}}{e \boldsymbol{B}}
$$

where $m_{\mathrm{i}}$ is the mass of the proton; $\bar{U}_{\mathrm{i}}$ is the mean thermal speed of the proton; and $e$ is the electric charge of the electron. Assuming that the $z$-extension of the domain of the non-adiabatic motion of the electrons (i.e., the order of electron gyroradius) is

$$
a_{\mathrm{e}}=\frac{m_{\mathrm{e}} \overline{U_{\mathrm{e}}}}{e \boldsymbol{B}}
$$

where $m_{\mathrm{e}}$ is the mass of the electron and $\overline{U_{\mathrm{e}}}$ is the mean thermal speed of electrons. Then, the total number of ions passing through the domain of the non-adiabatic motion of the ions should be

$$
\Delta N_{\mathrm{i}}=n \Delta x \Delta y a_{\mathrm{i}} \frac{\Delta t}{\tau_{\mathrm{i}}}
$$

where

$$
\tau_{\mathrm{i}}=\frac{\pi r_{\mathrm{i}}}{\overline{U_{\mathrm{i}}}}
$$

is the time an ion will stay in the domain of the non-adiabatic motion of the ions, or the half-ion cyclotron (in the $x y$-plane) period, and

$$
r_{i}=\frac{m_{i} \overline{U_{i}}}{e B_{z}}
$$

is the ion gyroradius in the finite $z$-directional magnetic field, $B_{z}$. Substituting Equations (A1), (A4), and (A5) into (A3), we obtain:

$$
\Delta N_{\mathrm{i}}=n \Delta x \Delta y \Delta t \frac{\overline{U_{i}}}{\pi} \frac{B_{z}}{B} .
$$

Likewise, the total number of electrons passing through the domain of the non-adiabatic motion of the electrons should be

$$
\Delta N_{\mathrm{e}}=n \Delta x \Delta y a_{\mathrm{e}} \frac{\Delta t}{\tau_{\mathrm{e}}}
$$

where

$$
\tau_{\mathrm{e}}=\frac{\pi r_{\mathrm{e}}}{\overline{U_{\mathrm{e}}}}
$$

is the time an electron will stay in the domain of the non-adiabatic motion of the electron or the half-electron cyclotron (in the $x y$ plane) period, and

$$
r_{\mathrm{e}}=\frac{m_{\mathrm{e}} \overline{U_{\mathrm{e}}}}{e B_{z}}
$$

is the electron gyroradius in the finite $z$-directional magnetic field $B_{z}$. Substituting Equations (A2), (A8), and (A9) into (A7), we obtain

$$
\Delta N_{\mathrm{e}}=n \Delta x \Delta y \Delta t \frac{\overline{U_{\mathrm{e}}}}{\pi} \frac{B_{z}}{B} .
$$

However, the particles have an equal chance of passing through the upper or lower portions of the flux tube. For simplicity, we assume that the particles move back to their original location (the same argument applies to the lower part of the X-line). Each ion will have an energy increment through the domain of the nonadiabatic motion of

$$
\Delta w_{\mathrm{i}}=2 r_{\mathrm{i}} E e,
$$

and each electron will have an energy increment through the domain of the non-adiabatic motion of

$$
\Delta w_{\mathrm{e}}=2 r_{\mathrm{e}} E e .
$$

The total energy increment of the ions through the domain of non-adiabatic motion will be

$$
\Delta W_{\mathrm{i}}=\Delta N_{\mathrm{i}} \Delta W_{\mathrm{i}}
$$

Substituting Equations (A5), (A6), and (A11) into (A13), we obtain

$$
\Delta W_{\mathrm{i}}=2 n \Delta x \Delta y \Delta t E \frac{m_{\mathrm{i}} \bar{U}_{\mathrm{i}}^{2}}{\pi B},
$$

where

$$
\overline{U_{i}}=\sqrt{\frac{8 k T_{i}}{\pi m_{i}}}
$$

and $k$ is Boltzmann's constant. Substituting Equation (A15) into (A14), we obtain:

$$
\Delta W_{\mathrm{i}}=16 n \Delta x \Delta y \Delta t E \frac{k T_{\mathrm{i}}}{\pi^{2} B}
$$

The total energy increment of the electrons through the domain of non-adiabatic motion is

$$
\Delta W_{\mathrm{e}}=\Delta N_{\mathrm{e}} \Delta w_{\mathrm{e}}
$$

Substituting Equations (A9), (A10), and (A12) into (A17), we ob- 
tain:

$$
\Delta W_{\mathrm{e}}=2 n \Delta x \Delta y \Delta t E \frac{m_{\mathrm{e}} \bar{U}_{\mathrm{e}}^{2}}{\pi B}
$$

where

$$
\overline{U_{\mathrm{e}}}=\sqrt{\frac{8 k T_{\mathrm{e}}}{\pi m_{\mathrm{e}}}} .
$$

Substituting Equation (A19) into (A18), we obtain

$$
\Delta W_{\mathrm{e}}=16 n \Delta x \Delta y \Delta t E \frac{k T_{\mathrm{e}}}{\pi^{2} B} .
$$

Let the total number of ions or electrons be $N$ in the upper part of the flux tube. Then, the total energy of the ions before reconnection is

$$
W_{\mathrm{i}}=\frac{3}{2} N k T_{\mathrm{i}}
$$

and the total energy of the electrons before reconnection is

$$
W_{\mathrm{e}}=\frac{3}{2} N k T_{\mathrm{e}}
$$

The $T_{\mathrm{i}} / T_{\mathrm{e}}$ ratio in the flux tube after reconnection will then be

$$
\frac{T_{\mathrm{i}, \mathrm{a}}}{T_{\mathrm{e}, \mathrm{a}}}=\frac{W_{\mathrm{i}}+\Delta W_{\mathrm{i}}}{W_{\mathrm{e}}+\Delta W_{\mathrm{e}}}=\frac{\frac{3}{2} N k T_{\mathrm{i}}+16 n \Delta x \Delta y \Delta t E \frac{k T_{\mathrm{i}}}{\pi^{2} B}}{\frac{3}{2} N k T_{\mathrm{e}}+16 n \Delta x \Delta y \Delta t E \frac{k T_{\mathrm{e}}}{\pi^{2} B}}=\frac{T_{\mathrm{i}}}{T_{\mathrm{e}}},
$$

where subscript a represents the time after reconnection.

An examination of Equation (A23) is necessary to understand the preservation or variation of the $T_{\mathrm{i}} / T_{\mathrm{e}}$ ratio. Equation (A23) indicates that the $T_{\mathrm{i}} / T_{\mathrm{e}}$ ratio is preserved after a patchy magnetic reconnection. However, this is only true when the temperatures of both the ions and electrons in Equations (A16) and (A20) are constant during the patchy reconnection. This condition does not hold once the accelerated particles return to the same domain of the non-adiabatic motion from the mirror point. In this case, Equation (A16) becomes:

$$
\Delta W_{\mathrm{i}}=16 n \Delta x \Delta y E \frac{k}{\pi^{2} B}\left(T_{\mathrm{i}} \tau_{\mathrm{i}}+T_{\mathrm{i}} t_{\mathrm{i}, \text { roundtrip }, 1}+T_{\mathrm{i}, 1} \tau_{\mathrm{i}}+T_{\mathrm{i}, 1} t_{\mathrm{i}, \text { roundtrip, } 2}+\cdots\right),
$$

where

$$
t_{i, \text { roundtrip }, I}=2 \frac{L}{\overline{U_{i}, l}}
$$

is the round-trip traveling time of the ions at distance $L$ from the reconnection site to the mirror point, and

$$
\overline{U_{i}, l}=\sqrt{\frac{8 k T_{\mathrm{i}, l}}{\pi m_{\mathrm{i}}}},
$$

where

$$
T_{\mathrm{i}, I}=T_{\mathrm{i},(I-1)}+\frac{4 r_{\mathrm{i},(I-1)} E e}{3 k}
$$

and subscript / represents the /th time non-adiabatic acceleration.

Likewise, Equation (A20) becomes
$\Delta W_{\mathrm{e}}=16 n \Delta x \Delta y E \frac{k}{\pi^{2} B}\left(T_{\mathrm{e}} \tau_{\mathrm{e}}+T_{\mathrm{e}} t_{\mathrm{e}, \text { roundtrip }, 1}+T_{\mathrm{e}, 1} \tau_{\mathrm{e}}+T_{\mathrm{e}, 1} t_{\mathrm{e}, \text { roundtrip }, 2}+\cdots\right)$

where

$$
t_{\mathrm{e}, \text { roundtrip }, l}=2 \frac{L}{\overline{U_{\mathrm{e}, l}}}
$$

is the round-trip time of the electron and

$$
\overline{U_{\mathrm{e}, l}}=\sqrt{\frac{8 k T_{\mathrm{e}, l}}{\pi m_{\mathrm{e}}}},
$$

where

$$
T_{\mathrm{e}, l}=T_{\mathrm{e},(l-1)}+\frac{4 r_{\mathrm{e},(l-1)} E e}{3 k}
$$

Then, the $T_{\mathrm{i}} / T_{\mathrm{e}}$ ratio will be

$$
\frac{T_{\mathrm{i}, \mathrm{a}}}{T_{\mathrm{e}, \mathrm{a}}}=\frac{W_{\mathrm{i}}+\Delta W_{\mathrm{i}}}{W_{\mathrm{e}}+\Delta W_{\mathrm{e}}} \neq \frac{T_{\mathrm{i}}}{T_{\mathrm{e}}}
$$

It should be noted that because the number density $n, x$-extension of the cross section of the flux tube $\Delta x$, and X-line length $\Delta y$ appear both explicitly or implicitly in the numerator and denominator of Equations (A23) and (A32), the $T_{\mathrm{i}} / T_{\mathrm{e}}$ ratios calculated by these two equations are independent of the quantities $n, \Delta x$, and $\Delta y$.

Here, charged particles are the particles inside a closed magnetic flux tube that undergoes magnetic reconnection. Some particles may not have the opportunity to reach the reconnection site and be accelerated if the reconnection time is too short or the distance between the location of the particle and the reconnection site is too large.

\section{References}

Angelopoulos, V., Kennel, C. F., Coroniti, F. V., Pellat, R., Kivelson, M. G., Walker, R. J., Russell, C. T., Baumjohann, W., Feldman, W. C., and Gosling, J. T. (1994). Statistical characteristics of bursty bulk flow events. J. Geophys. Res. Space Phys., 99(A11), 21257-21280. https://doi.org/10.1029/94JA01263

Baumjohann, W., Paschmann, G., and Cattell, C. A. (1989). Average plasma properties in the central plasma sheet. J. Geophys. Res. Space Phys., 94(A6), 6597-6606. https://doi.org/10.1029/JA094iA06p06597

Borovsky, J. E., Thomsen, M. F., Elphic, R. C., Cayton, T. E., and McComas, D. J. (1998). The transport of plasma sheet material from the distant tail to geosynchronous orbit. J. Geophys. Res. Space Phys., 103(A9), 20297-20331. https://doi.org/10.1029/97JA03144

Büchner, J., and Zelenyi, L. M. (1989). Regular and chaotic charged particle motion in magnetotaillike field reversals: 1. Basic theory of trapped motion. J. Geophys. Res. Space Phys., 94(A9), 11821-11842. https://doi.org/10.1029/JA094iA09p11821

Chen, C. X., and Wolf, R. A. (1999). Theory of thin-filament motion in Earth's magnetotail and its application to bursty bulk flows. J. Geophys. Res. Space Phys., 104(A7), 14613-14626. https://doi.org/10.1029/1999JA900005

Chen, C. X. (2013). Theoretical constraints on the cross-tail width of bursty bulk flows. Ann. Geophys., 31(12), 2179-2192. https://doi.org/10.5194/angeo-312179-2013

Chen, C. X., and Wang, C. P. (2019). Contribution of patchy reconnection to the ion-to-electron temperature ratio in the Earth's magnetotail. Earth Planet. Phys., 3(6), 474-480. https://doi.org/10.26464/epp2019049

Echim, M. M., and Lemaire, J. F. (2000). Laboratory and numerical simulations of the impulsive penetration mechanism. Space Sci. Rev., 92(3-4), 565-601. https://doi.org/10.1023/A:1005264212972 
Faganello, M., Califano, F., Pegoraro, F., Andreussi, T., and Benkadda, S. (2012). Magnetic reconnection and Kelvin-Helmholtz instabilities at the Earth's magnetopause. Plasma Phys. Control. Fusion, 54(12), 124037. https://doi.org/10.1088/0741-3335/54/12/124037

Hardy, D. A., Gussenhoven, M. S., and Brautigam, D. (1989). A statistical model of auroral ion precipitation. J. Geophys. Res. Space Phys., 94(A1), 370-392. https://doi.org/10.1029/JA094iA01p00370

Hasegawa, A., and Mima, K. (1978). Anomalous transport produced by kinetic Alfvén wave turbulence. J. Geophys. Res. Space Phys., 83(A3), 1117-1123. https://doi.org/10.1029/JA083iA03p01117

Hill, T. W. (1975). Magnetic merging in a collisionless plasma. J. Geophys. Res., 80(34), 4689-4699. https://doi.org/10.1029/JA080i034p04689

Izutsu, T., Hasegawa, H., Nakamura, T. K. M., and Fujimoto, M. (2012). Plasma transport induced by kinetic Alfvén wave turbulence. Phys. Plasmas, 19(10), 102305. https://doi.org/10.1063/1.4759167

Johnson, J. R., and Cheng, C. Z. (1997). Kinetic Alfvén waves and plasma transport at the magnetopause. Geophys. Res. Lett., 24(11), 1423-1426. https://doi.org/10.1029/97GL01333

Johnson, J. R., and Wing, S. (2009). Northward interplanetary magnetic field plasma sheet entropies. J. Geophys. Res. Space Phys., 114(A9), A00D08. https://doi.org/10.1029/2008JA014017

Lavraud, B., Thomsen, M. F., Lefebvre, B., Schwartz, S. J., Seki, K., Phan, T. D., Wang, Y. L., Fazakerley, A., Rème, H., and Balogh, A. (2006). Evidence for newly closed magnetosheath field lines at the dayside magnetopause under northward IMF. J. Geophys. Res. Space Phys., 111 (A5), A05211. https://doi.org/10.1029/2005JA011266

Lavraud, B., Borovsky, J. E., Génot, V., Schwartz, S. J., Birn, J., Fazakerley, A. N., Dunlop, M. W., Taylor, M. G. G. T., Hasegawa, H., ... Wild, J. A. (2009). Tracing solar wind plasma entry into the magnetosphere using ion-to-electron temperature ratio. Geophys. Res. Lett., 36(18), L18109. https://doi.org/10.1029/2009GL039442

Li, W., André, M., Khotyaintsev, Y. V., Vaivads, A., Graham, D. B., ToledoRedondo, S., Norgren, C., Henri, P., Wang, C., ... Strangeway, R. J. (2016). Kinetic evidence of magnetic reconnection due to Kelvin-Helmholtz waves. Geophys. Res. Lett., 43(11), 5635-5643. https://doi.org/10.1002/2016GL069192

Nakamura, R., Baumjohann, W., Mouikis, C., Kistler, L. M., Runov, A., Volwerk, M., Asano, Y., Vörös, Z., Zhang, T. L., ... Balogh, A. (2004). Spatial scale of highspeed flows in the plasma sheet observed by Cluster. Geophys. Res. Lett., 31(9), L09804. https://doi.org/10.1029/2004GL019558

Nykyri, K., Otto, A., Lavraud, B., Mouikis, C., Kistler, L. M., Balogh, A., and Rème, H. (2006). Cluster observations of reconnection due to the Kelvin-Helmholtz instability at the dawnside magnetospheric flank. Ann. Geophys., 24(10), 2619-2643. https://doi.org/10.5194/angeo-24-2619-2006

Onsager, T. G., Thomsen, M. F., Elphic, R. C., and Gosling, J. T. (1991). Model of electron and ion distributions in the plasma sheet boundary layer. J. Geophys. Res. Space Phys., 96(A12), 20999-21011. https://doi.org/10.1029/91JA01983
Onsager, T. G., Scudder, J. D., Lockwood, M., and Russell, C. T. (2001). Reconnection at the high-latitude magnetopause during northward interplanetary magnetic field conditions. J. Geophys. Res. Space Phys., 106(A11), 25467-25488. https://doi.org/10.1029/2000JA000444

Otto, A., and Fairfield, D. H. (2000). Kelvin-Helmholtz instability at the magnetotail boundary: MHD simulation and comparison with Geotail observations. J. Geophys. Res. Space Phys., 105(A9), 21175-21190. https://doi.org/10.1029/1999JA000312

Paschmann, G., Baumjohann, W., Sckopke, N., Phan, T. D., and Lühr, H. (1993). Structure of the dayside magnetopause for low magnetic shear. J. Geophys. Res. Space Phys., 98(A8), 13409-13422. https://doi.org/10.1029/93JA00646

Phan, T. D., Paschmann, G., Baumjohann, W., Sckopke, N., and Lühr, H. (1994). The magnetosheath region adjacent to the dayside magnetopause: AMPTE/IRM observations. J. Geophys. Res. Space Phys., 99(A1), 121-141. https://doi.org/10.1029/93JA02444

Schriver, D., Ashour-Abdalla, M., and Richard, R. L. (1998). On the origin of the ion-electron temperature difference in the plasma sheet. J. Geophys. Res. Space Phys., 103(A7), 14879-14895. https://doi.org/10.1029/98JA00017

Slavin, J. A., Smith, E. J., Sibeck, D. G., Baker, D. N., Zwickl, R. D., and Akasofu, S. I. (1985). An ISEE 3 study of average and substorm conditions in the distant magnetotail. J. Geophys. Res. Space Phys., 90(A11), 10875-10895. https://doi.org/10.1029/JA090iA11p10875

Song, P., and Russell, C. T. (1992). Model of the formation of the low-latitude boundary layer for strongly northward interplanetary magnetic field. J. Geophys. Res. Space Phys., 97(A2), 1411-1420. https://doi.org/10.1029/91JA02377

Song, P., DeZeeuw, D. L., Gombosi, T. I., Groth, C. P. T., and Powell, K. G. (1999). A numerical study of solar wind-magnetosphere interaction for northward interplanetary magnetic field. J. Geophys. Res. Space Phys., 104(A12), 28361-28378. https://doi.org/10.1029/1999JA900378

Tsyganenko, N. A. (1995). Modeling the Earth's magnetospheric magnetic field confined within a realistic magnetopause. J. Geophys. Res. Space Phys., 100(A4), 5599-5612. https://doi.org/10.1029/94JA03193

Tsyganenko, N. A., and Mukai, T. (2003). Tail plasma sheet models derived from Geotail particle data. J. Geophys. Res. Space Phys., 108(A3), 1136. https://doi.org/10.1029/2002JA009707

Wang, C. P., Lyons, L. R., Nagai, T., Weygand, J. M., and Lui, A. T. Y. (2010). Evolution of plasma sheet particle content under different interplanetary magnetic field conditions. J. Geophys. Res. Space Phys., 115(A6), A06210. https://doi.org/10.1029/2009JA015028

Wang, C. P., Gkioulidou, M., Lyons, L. R., and Angelopoulos, V. (2012). Spatial distributions of the ion to electron temperature ratio in the magnetosheath and plasma sheet. J. Geophys. Res. Space Phys., 117(A8), A08215. https://doi.org/10.1029/2012JA017658

Wing, S., Johnson, J. R., Chaston, C. C., Echim, M., Escoubet, C. P., Lavraud, B., Lemon, C., Nykyri, K., Otto, A., ... Wang, C. P. (2014). Review of solar wind entry into and transport within the plasma sheet. Space Sci. Rev., 184(1-4), 33-86. https://doi.org/10.1007/s11214-014-0108-9 